\title{
1 Quantitative Systems Pharmacology Modeling of PBMC-humanized Mouse to Facilitate \\ 2 Preclinical Immuno-Oncology Drug Development
}

3 Huilin $\mathrm{Ma}^{1 *}$, Minu Pilvankar ${ }^{3}$, Jun Wang ${ }^{3}$, Craig Giragossian ${ }^{3}$, Aleksander S. Popel $^{1,2}$

$4{ }^{1}$ Department of Biomedical Engineering, Johns Hopkins University School of Medicine,

5 Baltimore, MD, USA

$6 \quad{ }^{2}$ Department of Oncology and Sidney Kimmel Comprehensive Cancer Center, Johns Hopkins

7 University, Baltimore, MD, USA

$8{ }^{3}$ Biotherapeutics Discovery Research, Boehringer Ingelheim Pharmaceuticals, Inc, Ridgefield, 9 CT, USA

10 *Corresponding author email: huilin.ma2019@gmail.com

\section{Contents}

13 - Figure S1 Simulated 4-compartment, 2-compartment and measured cibisatamab mouse plasma 14 concentration (pg. S-2)

15 - Figure S2 Model predictions for in vivo tumor growth in NOG mice (pg. S-3)

- Figure S3 Model predictions and observed data for in vitro cytokine concentration (pg. S-4)

- Figure S4 The partial rank correlation coefficient for individual parameters (pg. S-5)

- Figure S5 Intratumoral T cell density and number of CEACEA_TCE_CD3 trimers after i.p. transfer of human T cells (pg. S-7)

- Table S1 Abbreviations (pg. S-8)

- Table S2 Experimental T cell-positive tumor area and simulated T cell density (pg. S-9) 
28

29

30

31

B.
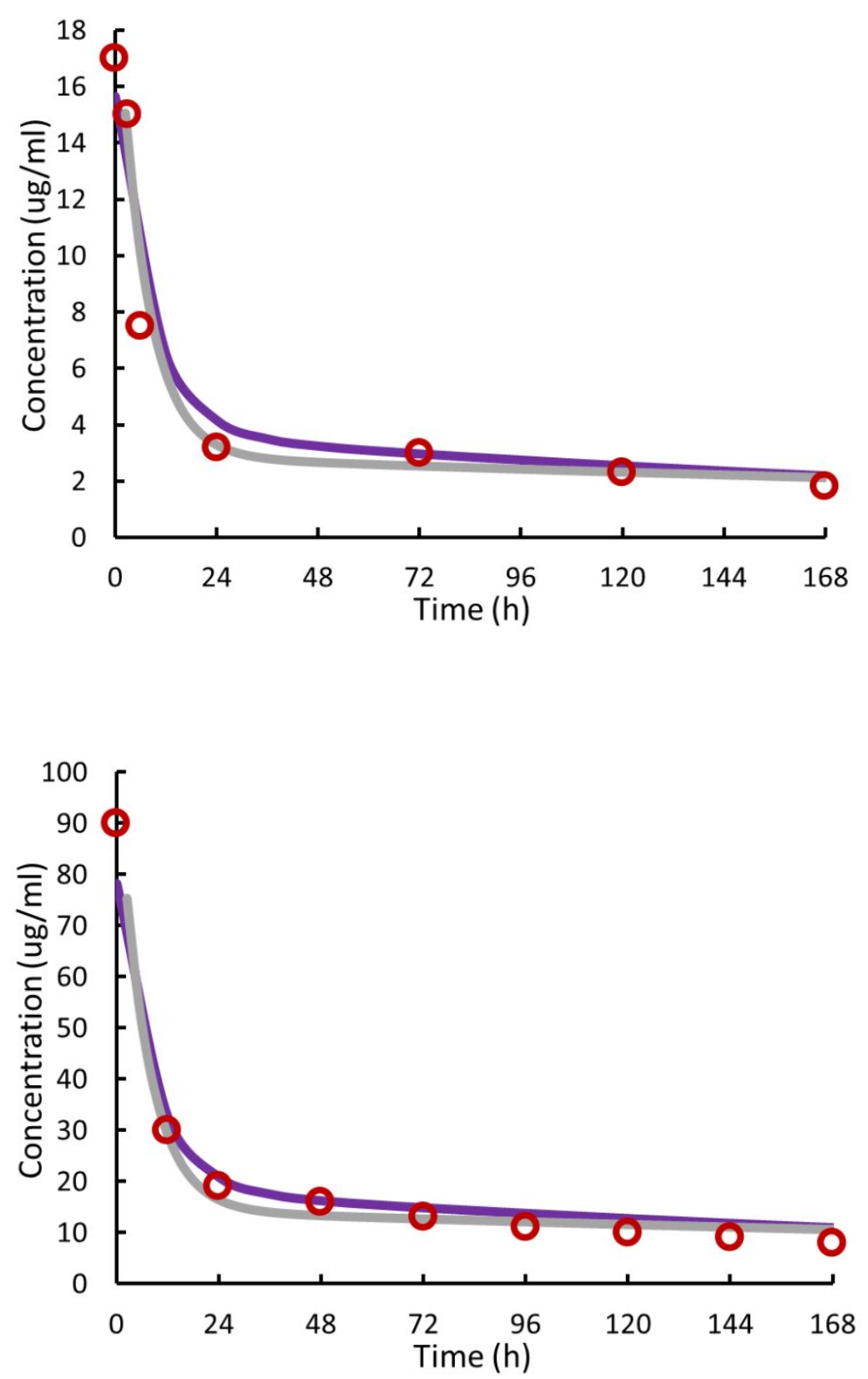
A.

33 Fig. S1 Simulated 4-compartment (solid purple lines), 2-compartment (solid grey

34 lines) and measured (red dots) cibisatamab mouse plasma concentration. A. 0.5

$35 \mathrm{mg} / \mathrm{kg} \mathrm{CEA-TCB,} \mathrm{i.v.} \mathrm{bolus.} \mathrm{B.} 2.5 \mathrm{mg} / \mathrm{kg} \mathrm{CEA-TCB,} \mathrm{i.v.} \mathrm{bolus.} \mathrm{Data} \mathrm{points} \mathrm{were}$ 36 digitized from Bacac et al. ${ }^{25}$. 


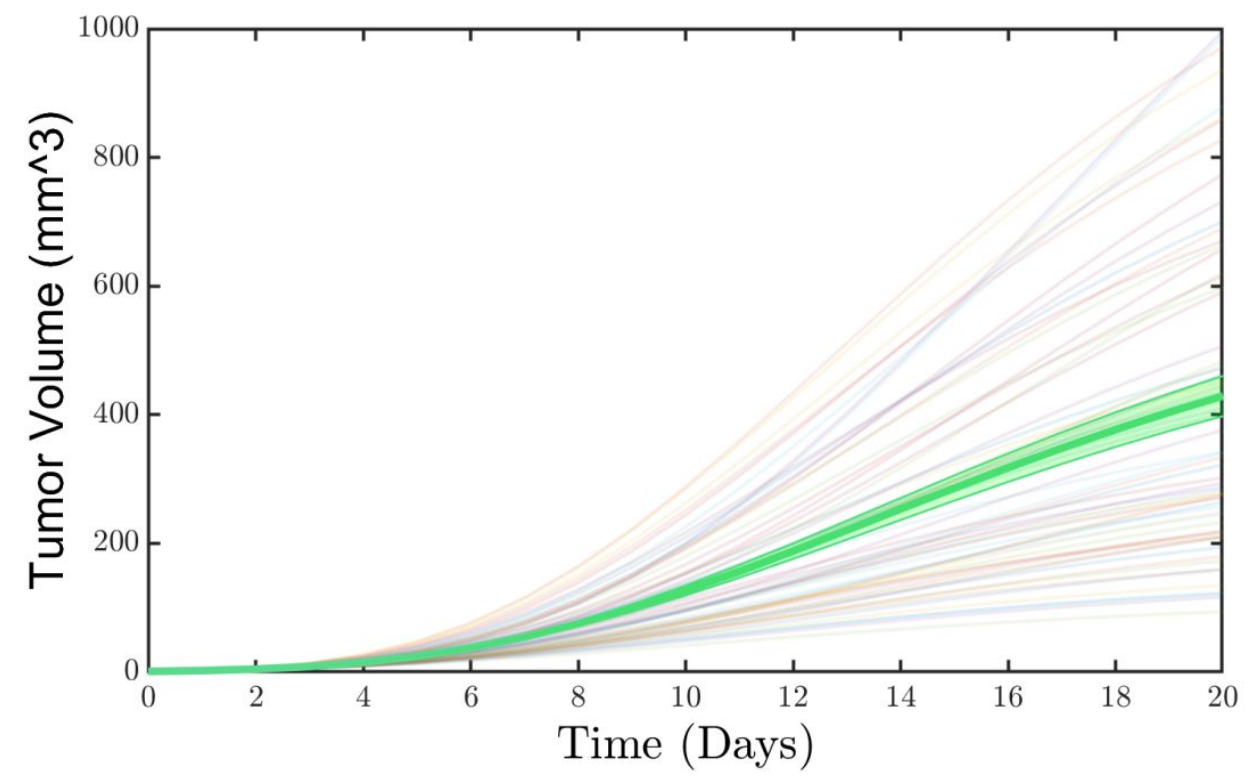

37

38 Fig. S2 Model predictions for in vivo tumor growth in NOG mice receiving i.p. 39 transfer of human $\mathrm{T}$ cells. Simulation results are shown as color solid lines and 40 presented by mean \pm SEM (solid green line and green shading, $\mathrm{n}=100$ ).

41

42

43

44

45

46

47

48

49

50

51

52 
A.

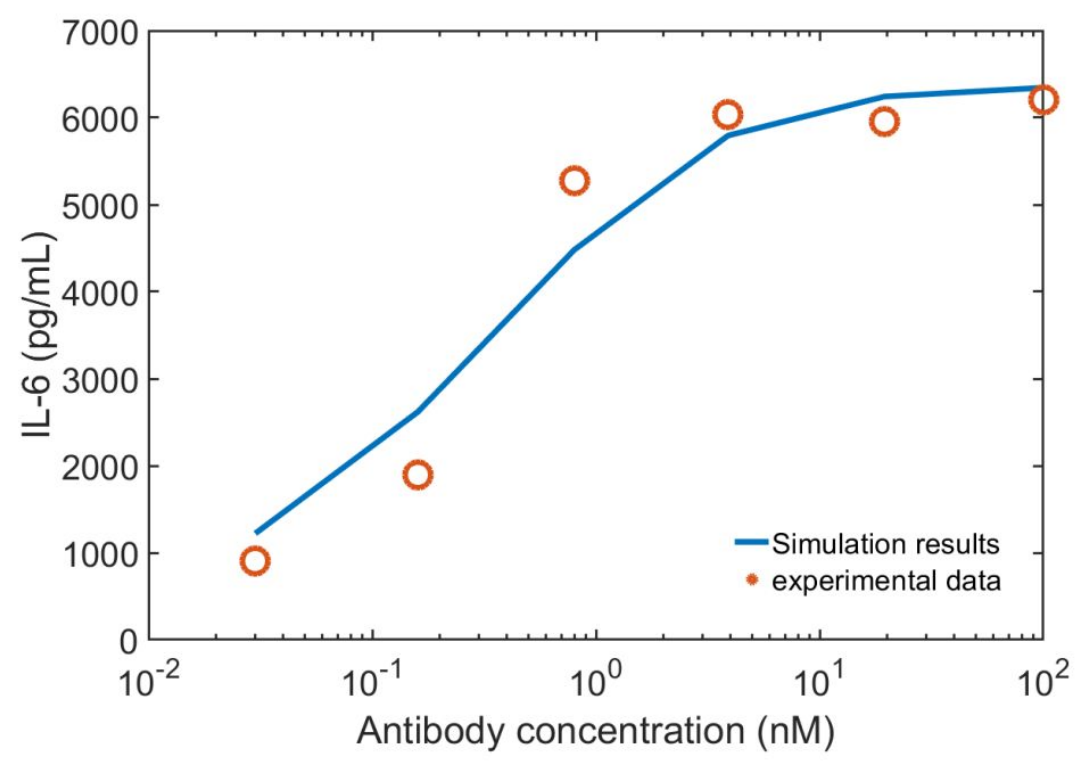

54

55 B.

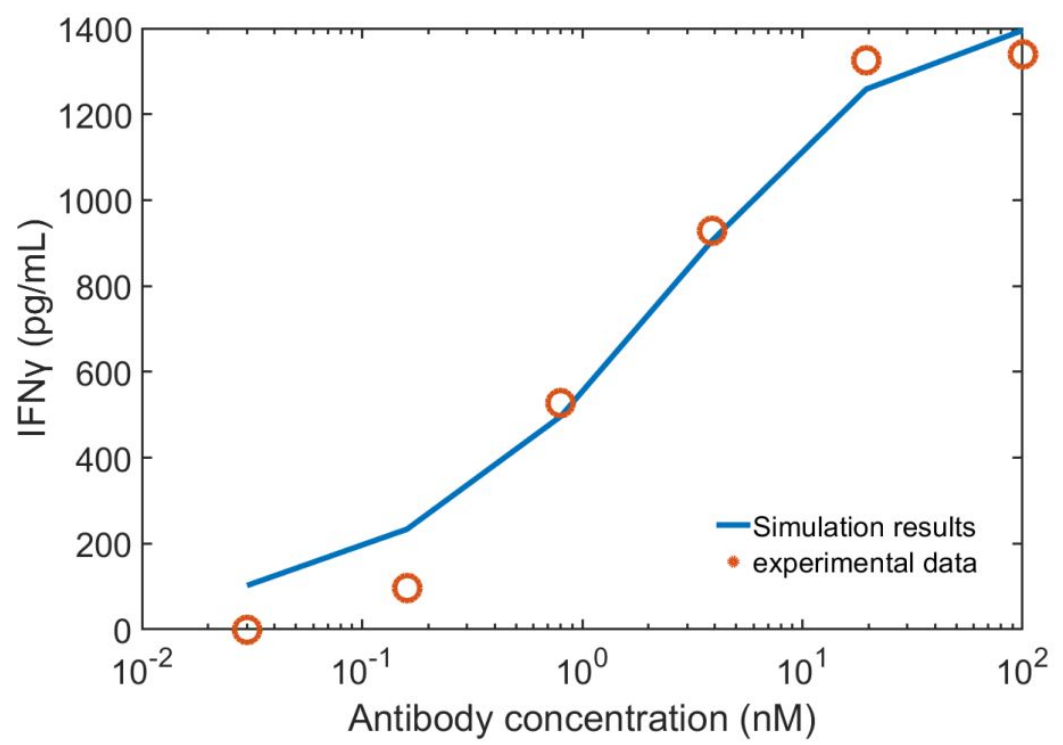

56

57 Fig. S3 Model predictions and observed data for in vitro cytokine concentration 58 with TCE treatment for IL-6 (A) and IFN $\gamma(\mathrm{B})$. Experimental data are plotted as 59 orange dots and simulation results are presented as solid blue line. Data points 60 were digitized from Bacac et al. ${ }^{25}$. 


\section{A.}

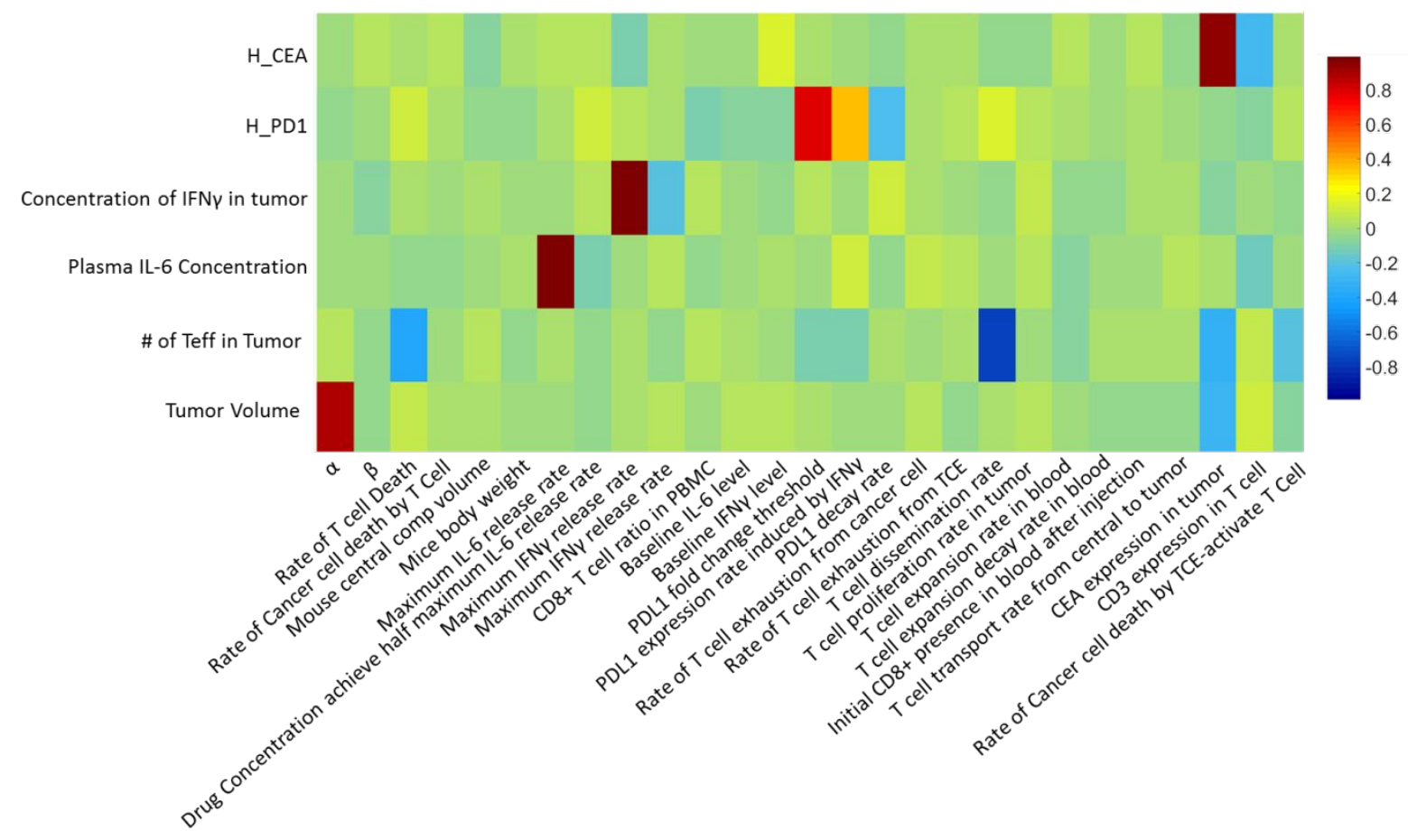

62

63 B.

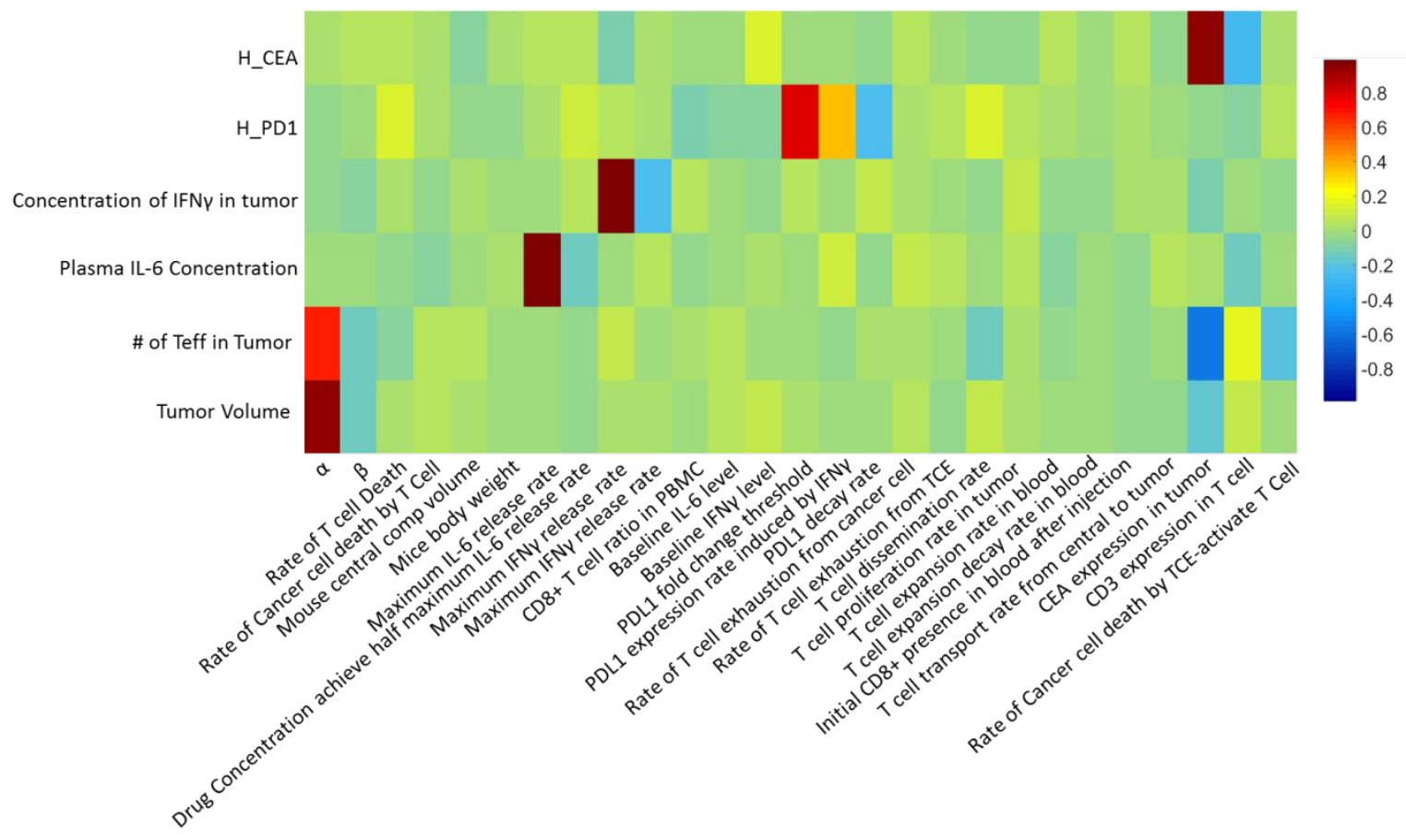


C.

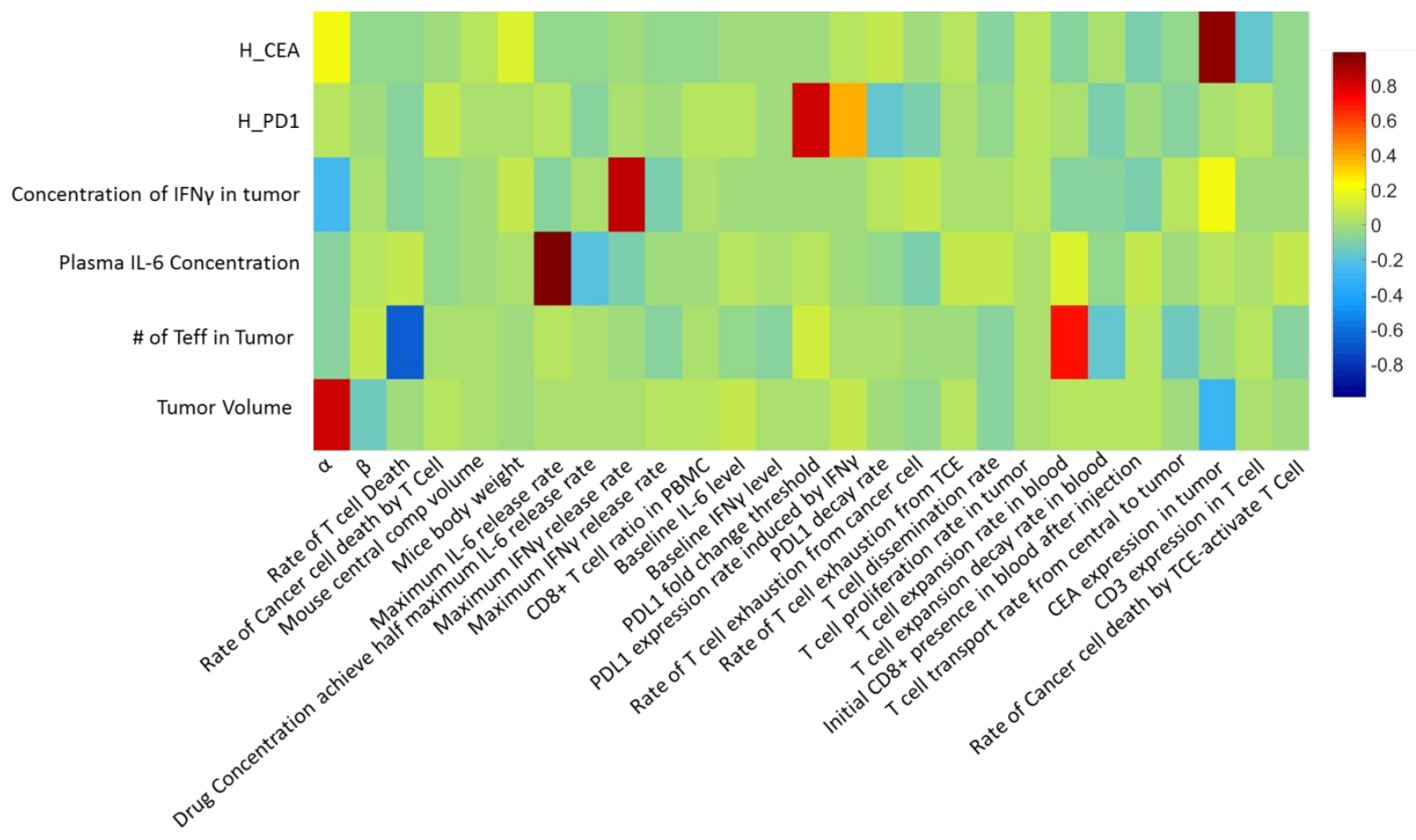

68 Fig. S4 The partial rank correlation coefficient, PRCC, for individual parameters

69 TCE treatment starting day 1 (A), day 7 (B) after co-grafting of human T cells and 70 cancer cells or Day 10 after i.p. transfer of human T cells (C).

71

72

73

74

75

76

77

78

79 
A.

81

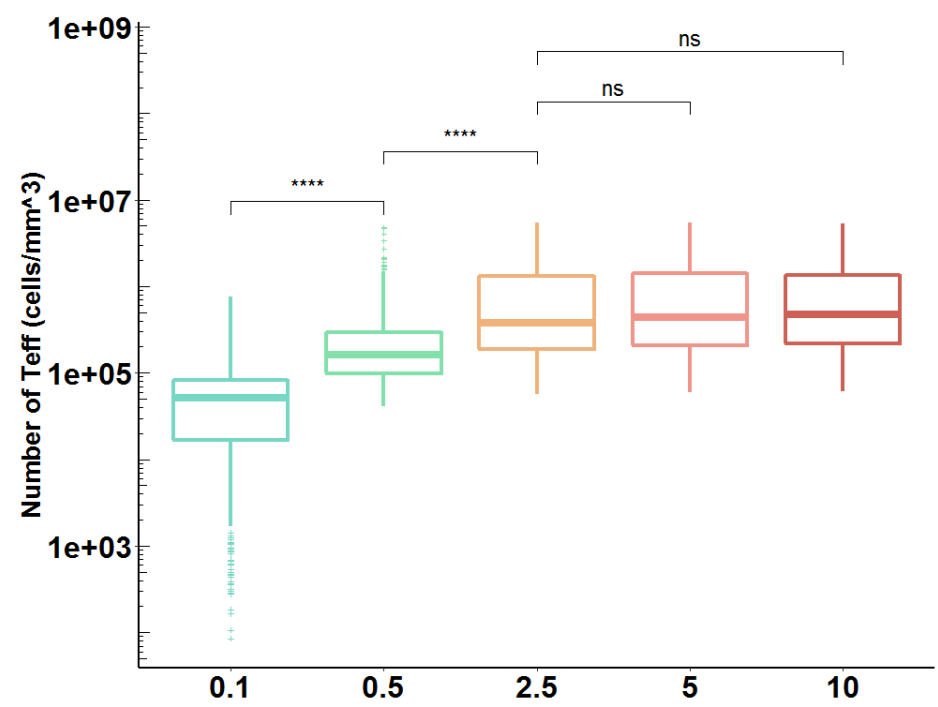

82 B.

83

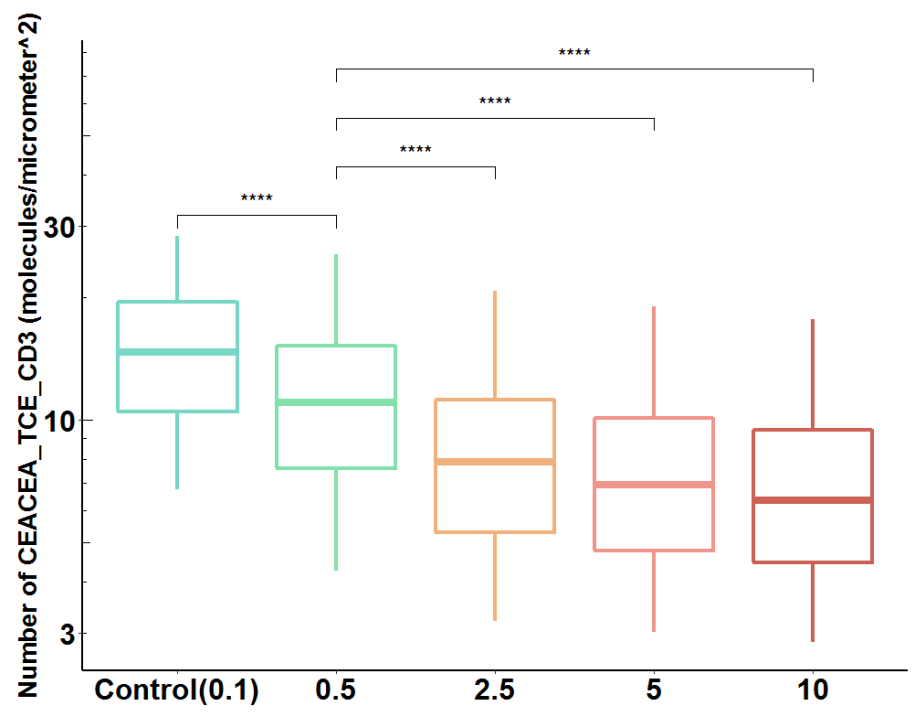

84 Fig. S5 Intratumoral T cell density (A) and number of CEACEA_TCE_CD3

85 trimers (B) for Day 10 after i.p. transfer of human T cells. 5 doses were

86 investigated and compared $(0.1,0.5,2.5,5$ or $10 \mathrm{mg} / \mathrm{kg}$, i.v., twice/week).

87 


\section{Supplementary Table}

89 Table. S1 Abbreviations

\begin{tabular}{|c|c|}
\hline Abbreviation & Definition \\
\hline CEA & Carcinoembryonic Antigen \\
\hline CEA-TCB & Carcinoembryonic Antigen T-Cell Bispecific Antibody \\
\hline HSC & hematopoietic stem cells \\
\hline MoA & mechanism of action \\
\hline NSCLC & Non-small-cell Lung Carcinoma \\
\hline PBMC & Peripheral Blood Mononuclear Cell \\
\hline PD-1 & Programmed cell death protein 1 \\
\hline PD-L1 & Programmed death-ligand 1 \\
\hline PSA & Parameter Sensitivity Analysis \\
\hline QSP & Quantitative Systems Pharmacology \\
\hline SBML & Systems Biology Markup Language \\
\hline TCB & T Cell Bispecific \\
\hline TCE & $\mathrm{T}$ cell engager \\
\hline TdLN & Tumor-draining Lymph Nodes \\
\hline Teff & effector $\mathrm{T}$ cell \\
\hline BsAbs & bispecific antibodies \\
\hline ICIs & immune checkpoint inhibitors \\
\hline GvHD & Graft-versus-host disease \\
\hline $\mathrm{PK} / \mathrm{PD}$ & Pharmacokinetics and Pharmacodynamics \\
\hline ODEs & ordinary differential equations \\
\hline CRS & cytokine release syndrome \\
\hline LHS & Latin Hypercube Sampling \\
\hline irAE & immune-related adverse events \\
\hline $\mathrm{MHC}$ & major histocompatibility complex \\
\hline
\end{tabular}


96 Table. S2 Experimental T cell-positive tumor area obtained from Lehmann et al. ${ }^{26}$ and simulated 97 T cell density at Day 4 and 5 in Figure. 2A and 2B

\begin{tabular}{ccccc}
\hline Experiment & Time & Mean Area & Mean \pm SEM & Day5/Day4 \\
\hline Control & Day 4 & 0.065 & $(0.052,0.078)$ & $\mathbf{0 . 6 6}(\mathbf{0 . 4 6 , 0 . 9 6 )}$ \\
& Day 5 & 0.043 & $(0.036 .0 .05)$ & \\
CEA TCB & Day 4 & 0.041 & $(0.032,0.05)$ & $4.53(3.28,6.53)$ \\
& Day 5 & 0.186 & $(0.164 .0 .209)$ & Day5/Day4 \\
\hline Simulation & Time & \# of T cells & Mean \pm SEM & $\mathbf{0 . 5 2}(\mathbf{0 . 4 8 , 0 . 5 5 )}$ \\
\hline Control & Day 4 & 354380 & $(344792,363968)$ & \\
& Day 5 & 182960 & $(176726,189193)$ & $5.07(4.52,5.66)$ \\
\hline
\end{tabular}

98

99 Table. S3 Experimental intertumoral T cell density obtained from Griessinger et al. ${ }^{29}$ with a 2D$1003 \mathrm{D}$ conversion provided by $\mathrm{Mi}$ et al. ${ }^{31}$ and simulated $\mathrm{T}$ cell density in Figure. 2D

\begin{tabular}{ccccccc}
\hline Experiments & Mean & SEM & & Mean & SEM \\
\hline Control & $\begin{array}{c}\text { 2D density } \\
\left(\text { cells } / \mathrm{mm}^{\wedge} 2\right)\end{array}$ & 2.17 & 0.72 & $\begin{array}{c}\text { 3D density } \\
\left(\text { cells } / \mathrm{mm}^{\wedge} 3\right)\end{array}$ & 220.8955 & 73.29251 \\
CEA TCB & & 212 & $\mathbf{8 8 . 6}$ & & 21580.57 & 9019.05 \\
\hline Simulations & & Mean & SEM & & Mean & SEM \\
\hline Control & $\begin{array}{c}\text { 2D density } \\
\left(\text { cells } / \mathrm{mm}^{\wedge} 2\right)\end{array}$ & $/$ & $/$ & 3D density & $/$ & $/$ \\
CEA TCB & & 209 & 25 & & 21264 & 2551 \\
\hline
\end{tabular}

101

102

103

104

105

106

107

108

109

110

111

112

113

114

115

116 


\section{Supplementary Equations}

\section{2-D/3-D density of $\mathbf{T}$ cell conversion}

119 In computational models the cell concentrations are usually represented as volume density rather 120 than area density. However, most experiments measured area density of T cells. Mi et al. ${ }^{31}$ has 121 developed a equation to obtain the volume density from experimental measurements, 3D

122 numerical densities $(V d)$ can be estimated from 2D density $(A d)$ using the following equation:

$$
V d=\frac{A d}{t+D-2 h}
$$

125

where $t$ is the thickness of the section (typically $t=4.5-5 \mu \mathrm{m}$ ), $D$ is the diameter of stained cells (which are lymphocytes in the scope of this study, $D=5-7.1 \mu \mathrm{m}$ for T cells), and $h$ is the minimum height of detectable spherical cap (which can be derived from cellular segmentation algorithm parameters). In this equation, $2 h$ in the denominator accounts for loss of undetected parts of the cell, $A_{\text {crit }}$ is the minimum area detectable during the segmentation, typically $\sim 10 \mu \mathrm{m}^{2}$. 133 Using this equation, one could convert the 2D densities (in mm-2) to 3D densities (in mm-3) ${ }^{31}$. 